\title{
Conservatisme et Progressisme en éducation: approches critiques et éclairages internationaux
}

\author{
André D. Robert
}

Introduction

Des deux notions qui constituent le titre du dossier thématique ${ }^{1}$ présenté dans ce $\mathrm{n}^{\circ}$ de la Revista Lusófona de Educação, la première, "conservatisme», semble - toutes proportions gardées - la moins sujette à des relativisations et fluctuations historiques. Sa racine française "conserver» (du latin "conservare», "cum», intensif, et «servare»: garder, maintenir) désigne sans conteste l'action de maintenir en l'état, de ne pas changer. On peut néanmoins distinguer deux nuances inhérentes aux usages du verbe et des substantifs qui en sont dérivés, selon que l'on met l'accent sur une fonction de conservation qui, tout en procédant certes d'une volonté, ne ressortit pas prioritairement à une intention politique au sens partisan: ainsi le métier de "conservateur» de musée consiste d'abord, avant toute autre mission, à protéger les œuvres de toutes les altérations possibles et si besoin à les restaurer afin de pouvoir les exposer, aux meilleures conditions, dans leur authenticité originaire. Lorsqu'on parle au sens strict de "conservatisme" et de "conservateurs" dans l'arène politique, s'affiche une volonté délibérée de ne rien changer, rien réformer par rapport à l'état actuel de la société et, dans tous les contextes nationaux, il n'est a priori pas difficile d'identifier le personnel politique concerné. Si la Révolution française de 
1789 porte encore une signification universelle (De Francesco, 2018) en établissant une coupure radicale avec «l'Ancien Régime», l'interprétation qu'en donne dès 1790 Edmund Burke dans ses Reflections on the Revolution in France peut être considérée comme emblématique de toute position conservatrice: est récusée l'intention de changer l'ordre politique existant, qui plus est par des moyens révolutionnaires, et toute rupture de cet ordre est refusée en tant que processus destructeur, menace pour les libertés individuelles (Edmund Burke, 1790). Cette tradition conservatrice «burkienne» issue d'une répulsion envers toute idée de révolution est perpétuée de nos jours dans le monde anglo-saxon par exemple par Roger Scruton (Scruton, 1984).

En matière d'éducation scolaire, le problème se révèle plus difficile à traiter en fonction de la manière dont on définit son essence ou la finalité qui lui est assignée. $\mathrm{Si}$, comme le philosophe français Gaston Berger, on inscrit unilatéralement l'éducation dans l'idée que, toute culture étant résolument prospective ${ }^{2}$, et n'étant point «la stérile évocation de choses mortes, mais la découverte d'un élan qui se transmet à travers les générations[...] qui à la fois réchauffe et éclaire ", en privilégiant cet élan, ce "feu» (Berger, 1967), on tourne alors le dos à toute position de conservation et on choisit la création du nouveau, peut-être le «progrès» (mais est-ce toujours sûr?). En revanche, si, à l'instar de Hannah Arendt, on considère que la capacité créatrice de l'éducation, permettant à terme aux nouvelles générations de révéler leur potentiel de «nouvelleté» ${ }^{3}$, ne peut reposer que sur une première action, indispensable et fondamentale, de conservation, assurée par l'école, on est amené à dialectiser les relations entre conservation et renouvellement, création («progrès»?). «Etant donné que le monde est vieux, toujours plus vieux qu'eux [les enfants], le fait d'apprendre est inévitablement tourné vers le passé, sans tenir compte de la proportion de notre vie qui sera consacrée au présent» (Arendt, [1958], 1972, p. 250). La dimension politique (au sens philosophique cette fois) n'est évidemment pas absente de ces prises de position, délimitant sans doute deux camps possibles en éducation, l'un tourné vers le prospectif (sinon le progrès), l'autre plus délibérément conservateur (et explicitement critique du «progressivism» américain incarné par Dewey), encore que Hannah Arendt l'argumente au nom de la possibilité du renouvellement (sinon, là aussi, du progrès): «C'est [...] avec l'éducation que nous décidons si nous aimons assez nos enfants pour ne pas les rejeter de notre monde [...] ni leur enlever leur chance d'entreprendre quelque chose de neuf, quelque chose que nous n'avions pas prévu, mais les préparer d'avance à la tâche de renouveler un monde commun» (Arendt [1958], 1972, p. 252).

Quant à la notion de «progressisme», elle s'avère beaucoup plus polysémique et changeante selon les contextes, nationaux, historiques et politiques. Issue du siècle des Lumières, elle désigne d'abord globalement le fait que, en s'appuyant sur les ressources de l'intelligence et de la raison, l'humanité a constamment amélioré sa condition dans l'histoire, orientée du moins bien vers le mieux, ce qui peut signifier 
l'existence d'un «sens» de ladite histoire. Le constat global - encore celui-ci doit-il être relativisé selon les points de vue et les univers, comme s'emploient à le démontrer le relativisme et le postmodernisme contemporains - d'un progrès scientifique et technique incontestablement bénéfique aux humains au cours du temps a conduit à en effectuer la transposition vers un postulat de progrès social et politique quasi permanent, par-delà les vicissitudes des totalitarismes et autres retours à des formes barbares ou tyranniques, postulat dont plusieurs acteurs se disputent le monopole pour l'intégrer à leurs différentes visions du monde.

Le «progressivism» aux Etats-Unis (que le français traduit par progressisme), dont Roosevelt, Kennedy, voire Johnson, ont pu se réclamer, s'est évidemment frontalement opposé à la conception du progrès que développaient les communistes et l'URSS, particulièrement pendant la Guerre Froide. En France, il s'est agi d'une notion politique très évolutive. Dans les débuts de la III République (régime qui couvre la période 18791940), on a commencé à appeler "progressistes» les successeurs des «républicains modérés», c'est-à-dire - comme leur nom l'indiquait - des hommes politiques peu enclins à la réforme: «En réalité, être un républicain 'progressiste' signifie surtout n'être pas un 'radical'. Car les groupes [...] qui se disent radicaux constituent toujours une aile gauche du camp républicain» (Agulhon, 1990, p. 60). Les progressistes d'alors sont résolument anti-socialistes et il n'est pas exceptionnel qu'une partie (majoritaire) de ces progressistes votent avec la droite, même lorsque l'enjeu est de «défendre la République» comme lors de la présentation à la Chambre des députés du gouvernement Waldeck-Rousseau en juin 1899 (Agulhon, 1990, p. 104). Sous la IVe République (1946-1958), renversement de perspectives, ce sont les communistes au premier chef qui se réclament du progressisme et admettent dans cette mouvance certains compagnons de route, faisant parfois des alliances électorales avec des républicains dits «de progrès»; mais les socialistes revendiquent aussi le terme, avec des objectifs et des références différents; en général - et cela s'est poursuivi sous la Ve République (à partir de 1959) - la gauche se dit progressiste face à la droite conservatrice, bien que celle-ci, dans sa version modernisatrice, se réclame aussi du progrès. Aujourd'hui, dans le cadre de la nouvelle configuration politique, les libéraux du parti macronien se proclament seuls progressistes (partisans de la réforme économique visant à libérer les marchés) face à la gauche et la droite traditionnelles, rejetées uniformément dans le camp conservateur. On voit que, selon le contexte et le point de vue adopté, il est très difficile de stabiliser une position relativement à l'acception du terme «progressisme».

Qu'en est-il dans la sphère éducative? C'est, au-delà de ce qui a été esquissé plus haut à travers les propos de $\mathrm{G}$. Berger et $\mathrm{H}$. Arendt, ce que ce dossier s'efforce d'examiner, en faisant varier les périodes et les contextes nationaux, et en montrant les circulations qui peuvent exister entre progressisme et conservatisme, qu'on se place à l'intérieur des frontières du premier ou même dans le périmètre du second. 
Sébastien Alix montre certaines ambiguïtés du progressisme étatsunien incarné en la personne du philosophe et pédagogue pragmatiste John Dewey. Alors que de Iongue date, en France et ailleurs, ce progressisme pédagogique, et politique, est paré de nombreuses vertus, présenté comme un modèle de renouvellement éducatif émancipateur, il n'est pas exempt de préjugés, notamment raciaux; la représentation de la société démocratique qu'il véhicule approuve en fait implicitement l'ordre établi. Sans viser l'abaissement systématique d'une idole, l'analyse de ses ambiguïtés permet «de nuancer et de compléter la représentation qui en est souvent donnée sans pour autant chercher à réduire ou minorer la profondeur indiscutable d'élaboration de l'œuvre éducative deweyenne». A travers les figures de Irving Babitt et de Norman Foerster, c'est précisément à une critique du progressisme pédagogique de J. Dewey, historiquement située dans la première moitié du $X X^{e}$ siècle, donc antérieure à la critique d'Arendt, que s'est intéressée Dominique Ottavi. Le courant littéraire qu'ont animé ces deux auteurs s'est baptisé "Nouvel Humanisme» (New Humanism) et s'est délibérément placé en opposition au progressisme, adossé chez Dewey à une conception philosophique pragmatiste dénoncée comme réductionniste et naturaliste. Aussi bien Babitt que plus tard (à partir des années 1930) son élève Foerster plaident pour une réhabilitation de la littérature, de la critique littéraire et des Humanités conçues comme les meilleurs vecteurs d'une vraie formation (au sens de Bildung), contre la thématique de la seule adaptation aux besoins et de la pure coïncidence de l'éducation scolaire avec la vie telle qu'elle est. II n'est donc pas illégitime aujourd'hui de considérer que «la critique de certaines formes du progressivisme, et l'engagement en faveur de la conservation de la culture héritée du passé ne relèvent pas nécessairement du conservatisme».

Dans les mêmes années 1930, alors que l'économie et la société argentine ont été très sensiblement affectées par la crise, une des réponses données l'a été par la réforme scolaire élaborée, dans une optique nationaliste et utilitaire, en associant de manière très éclectique spiritualisme philosophique, psychologie expérimentale, pédagogie active et propagande patriotique. Les effets de cette réforme ayant eu une Iongue portée, Adrian Ascolani cherche à démêler les composantes de la réforme pouvant relever tour à tour du conservatisme ou du progressisme. L'historien repère une tension entre des éléments de rénovation et des éléments relevant de la tradition, la contradiction épistémologique ayant été plus ou moins résolue dans la pratique: "Le spiritualisme, le spontanéisme, le pédocentrisme, et la préparation à la vie se sont installés dans le discours institutionnel, et se sont renforcés dans les années suivantes. L'orientation centrale de l'enseignement primaire s'est fondée sur la tentative de combiner les principes de l'école active inspirée de John Dewey avec la théorie des capacités à survivre d'Herbert Spencer».

Joaquim Pintassilgo et Alda Namora au Portugal, Noëlle Monin en France se penchent chacun sur la figure d'un pédagogue progressiste marquant de leur pays. La 
vie du pédagogue portugais Sergio Niza (né en 1940) s'identifie largement au développement du mouvement MEM (Movimiento de la Escuela Moderna), se réclamant des idées et principes de la pédagogie Freinet. Implanté à partir des années 1960, ce courant s'affirme donc d'abord timidement sous la dictature salazariste, ce qui constitue un paradoxe de cohabitation relative entre une orientation idéologique résolument progressiste et un régime conservateur et réactionnaire (même si Niza, chercheur invité à I'IPN à Paris, n'assume une responsabilité directoriale à Lisbonne qu'à partir de 1973, à toute proximité temporelle de la Révolution des CEillets). C'est grâce à l'action pédagogique de Sergio Niza, forgée dans la lutte pour la démocratie et référée à la «tradition d'innovation" (Peter Burke, 2007), grâce à «son enracinement dans la pédagogie progressiste et, en particulier, à son effort permanent pour repenser les modèles» que les auteurs - eux-mêmes engagés pédagogiquement - estiment pouvoir aujourd'hui «continuer à regarder dans la perspective de l'innovation». Robert Gloton (1906-1986) a suivi le parcours classique d'un instituteur français formé à l'école normale (et même dans la plus célèbre d'entre elles, l'école normale d'Auteuil, à Paris) avant de devenir inspecteur primaire, c'est-à-dire responsable administratif et pédagogique des instituteurs d'une circonscription (un territoire délimité à l'intérieur d'un département). Ayant rejoint les idées du mouvement pédagogique progressiste GFEN (Groupe français d'éducation nouvelle), il a créé sur cette base le GCEN (Groupe coopératif d'éducation nouvelle), fondé une revue (Echanges) et prétendu faire de sa circonscription un petit laboratoire de l'innovation. A travers l'analyse de cette revue et des traces de la pratique de Gloton, Noëlle Monin considère qu'il n'a cependant pas été à l'abri de contradictions entre progressisme et conservatisme, notamment en maintenant les postures liées à sa position hiérarchique d'inspecteur, là où on aurait attendu un déplacement des rapports avec ses administrés, et surtout en esquivant une vision sociologique critique de l'école : «Son parti pris pour un enseignement en rupture partielle avec l'académisme institutionnel ne l'a pas amené néanmoins à se poser des questions sociologiques sur l'école et sa fonction de sélection sociale, ni à se demander s'il suffit d'innover pour changer l'école".

Le dossier se prolonge avec trois contributions relatives au contexte français, mais dont la portée peut être envisagée sous certains aspects comme extra-nationale, car touchant aux réactions vis-à-vis des réformes ou projets de réforme dans le monde scolaire. Yves Verneuil s'intéresse au syndicalisme enseignant du second degré autour des événements de mai 1968, montrant que, pour l'essentiel, il existe, à gauche (SNES, SGEN), une convergence entre progressisme politique et pédagogique et de même, à droite, entre conservatisme politique et pédagogique (SNALC). Or un nouveau venu vient troubler le jeu, la CNGA, certes minoritaire mais gagnant une petite influence pendant un court moment, en cherchant à se distinguer de son rival conservateur, le SNALC. Hostile à de nombreuses mesures pédagogiques prises après mai 1968, 
donc elle-même située à droite et conservatrice sur le plan politique, la CNGA entend cependant se proclamer réformatrice («réformiste»?) sur certains points, prenant ainsi acte - à la différence d'autres - du caractère inéluctable de l'enseignement de masse, et approuvant par exemple la création de foyers socio-éducatifs et l'idée d'équipes pédagogiques. "Avec la CNGA, on semble [...] avoir affaire à un syndicat enseignant conservateur du point de vue politique et social, mais réformiste du point de vue pédagogique», ce «réformisme» revendiqué devant néanmoins être interrogé de près.

Frédéric Mole et Pierre Kahn traitent, à deux moments historiques (tout début du $X X{ }^{e}$ siècle pour le premier, époque contemporaine pour le second) de la particularité de certains positionnements relatifs à l'enseignement de la langue française (orthographe, grammaire, littérature), donnant toujours matière à des débats assez dramatisés. Aux alentours de 1900, les partisans d'une réforme de l'orthographe, consistant en des tolérances sur quelques aspects litigieux de l'écriture de la langue, argumentent en disant que les instituteurs ont plus à valoriser, eu égard aux enjeux démocratiques, la dimension intellectuelle et morale de leur enseignement que le caractère formaliste de celui-ci, renvoyant ainsi les opposants à la réforme dans le camp des conservateurs. Ceux-ci, en la personne par exemple d'un rédacteur de la Revue de l'enseignement primaire, se surnommant emblématiquement Populo, contre-attaquent avec des arguments qui empruntent au registre démocratique et "progressiste»: "Priver les enfants du peuple d'un accès à la normativité de la langue (orthographique et syntaxique), ce n'est pas faire œuvre démocratique [...]». F. Mole avance l'expression de «conservateurs-progressistes» pour désigner ces acteurs pédagogiques, politiquement progressistes, qui ne se résolvent pas à l'idée condescendante d'un abaissement des exigences disciplinaires pour les élèves issus des catégories populaires, et à ce titre entendent défendre l'existant. C'est au fond le même type de schéma que retrouve Pierre Kahn lorsqu'il analyse les positions, près d'un siècle plus tard, de l'association «Sauver les lettres» fondée sur des bases «anti-pédagogistes» (accusant certains courants pédagogiques de vouloir liquider la culture et les Humanités) et conservatrices (voulant préserver les acquis de la tradition), tout en se réclamant du progressisme politique de la gauche. La difficulté venant de ce que ces positions coïncident en partie avec d'autres délibérément situées à droite, P. Kahn examine deux types d'explication de ce conservatisme scolaire de gauche (donc en principe cohabitant avec le progressisme), l'une en référence à l'idée d'émancipation héritée de Condorcet, l'autre, plus radicale, référée à une argumentation d'opposition aux ruses actuelles du capitalisme et s'appuyant sur l'«idée selon laquelle, pour que tout change (émancipation, frein à l'entreprise de domination mondiale du système capitaliste ...) il faut (même s'il ne suffit peut-être pas) que, dans le domaine de la culture et de l'enseignement, rien ne change ${ }^{4}$. 


\section{Notas}

1 Issu des travaux menés dans le cadre du Standing Working Group REFORPRO réuni à l'occasion des congrès de I'ISCHE (International Standing Conference of History of Education) en 2016 (Chicago), 2017 (Buenos Aires), 2018 (Berlin).

2 Le substantif «la prospective» fut forgé par Berger lui-même, au sens d'étude des futurs possibles.

3 Terme emprunté à Montaigne.

4 II s'agit là d'une sorte «d'effet-Guépard» inversé si l'on se souvient de la phrase prêtée à son jeune héros aristocrate Tancrède par G. Tomasi di Lampedusa dans son roman Le Guépard: «Pour que rien ne change, il faut que tout change» également citée par Y. Verneuil et P. Kahn dans leurs contributions.

\section{Bibliographie}

Agulhon, M. (1990). La République. 1880 à nos jours. Tome 5 de Histoire de France Hachette. Paris: Hachette.

Arendt, H. ([1958]1972). La crise de l'éducation in La crise de la culture. (pp. 223-252). Paris: Gallimard, coll. Idées.

Berger, G. (1967). L’homme moderne et son éducation. (2éd.). Paris: PUF.

Burke, E. (1790). Reflections on the Revolution in France and on the Proceedings in Certain Societies in London relative to that Event in a Letter intended to have been Sent to a Gentleman in Paris. Londres: J.Dodsley.

Burke, P. (2007). Cultura, tradição, educação. In D. Gatti Júnior \& J. Pintassilgo (Org.). Percursos e desafios da pesquisa e do ensino de História da Educação (pp. 13-22). Uberlândia: Edufu.

De Francesco, A. (2018). La guerre de deux cents ans. Une histoire des histoires de la Révolution française. Paris: Perrin.

Montaigne (de), M. ([1588]1992). Les Essais. Paris: Arléa.

Scruton, R. (1984). The meaning of Conservatism. (2éd.). London: MacMillan.

Tomasi di Lampedusa, G. ([1958]1959). Le Guépard. Paris: Seuil.

\section{André D. Robert}

Professeur émérite de sciences de l'éducation à l'université Louis Lumière Lyon 2 (France) et professeur régulièrement invité à I'Universidade Lusófona de Lisbonne. II travaille sur les politiques éducatives et les syndicalismes enseignants; il s'intéresse également aux transformations de la «forme scolaire», ainsi qu'aux questions d'épistémologie. Email: andre.robert@univ-lyon2.fr 